\title{
The Undulatory Changes in the Spleen Volume and Their Effects on the Blood Flow in the Splenic Vein
}

\author{
Takehiko Semba, Kazumoto Fujii and Nobumasa Kimura \\ Department of Physiology (Prof. T. Semba), \\ University of Hiroshima Medical School, Hiroshima
}

\begin{abstract}
The effect of rhythmical contraction of the spleen on the splenic venous flow was studied. The rate of blood flow through the splenic vein was determined in dogs which maintained an almost constant systemic blood pressure before and after the rhythmical contractions was induced. Normal splenic blood flow averaged $2.77 \mathrm{ml}$ per minute, but increased to $3.22 \mathrm{ml}$ per minute on the average during the period of rhythmical contractions. This increase corresponded to $16.2 \%$ of the normal splenic blood flow. This suggests that the rhythmical fluctuation in splenic blood flow accelerates the blood flow in the portal vein.
\end{abstract}

The rhythmical contraction of the spleen was first reported by Roy ${ }^{1}$ in 1881 , and subsequently detailed studies of this phenomenon were made by Schäfer and Moore $^{2}$. Barcroft and Nisimaru, ${ }^{3}$ studying the relationship between the rhythmical variations in the spleen volume and their effects on the arterial blood pressure, found that a change of one milliliter in the spleen volume brought about a change of $6 \mathrm{~mm} \mathrm{Hg}$ in arterial blood pressure. Semba, Fukuda and Kishi ${ }^{4}$ demonstrated that the contraction of the spleen with a reduction of $0.6 \mathrm{ml}$ in volume elevated the portal venous pressure by $7.6 \mathrm{~mm} \mathrm{H}_{2} \mathrm{O}$ which corresponded to $10.9 \%$ of the portal venous pressure. But the effect of rhythmical contractions of the spleen on the blood flow through the spleen has not yet been investigated.

The present paper deals with the correlation between the rhythmical contraction of the spleen, systemic blood pressure, and splenic venous pressure and venous flow.

\section{MethoDs}

In this study 31 dogs weighing about $12 \mathrm{~kg}$ were employed. They were anesthetized by intravenous injection of Nembutal (Abbott Co.) in a dose of $25 \mathrm{mg} / \mathrm{kg}$ body weight.

An oncometer was used for measuring the splenic volume. The blood pressure of the splenic vein and the systemic blood pressure of the common carotid artery were measured by electronic manometers (Nihon Kohden Co. MP-4).

Received for publication, August 15, 1966. 
For the measurement of the blood flow in the splenic vein, an electromagnetic flow-meter was employed (Nihon Kohden Co. MF-2). These were all recorded by use of an ink-writing oscillograph (Nihon Kohden Co. WI-180).

The undulatory changes in the carotid arterial pressure, splenic venous pressure, splenic volume and splenic venous flow were induced by intravenous injection of adrenaline, lobeline, defibrinated blood or saline solution, by bloodletting, by stimulation of the central end of the vagus or sciatic nerves, or by temporary suspension of the splenic circulation. ${ }^{4,5}$

\section{Results}

Simultaneous recording of the carotid arterial and splenic venous pressures, splenic volume and splenic venous flow was made in 25 out of 31 dogs. The typical undulatory changes are shown in Figs. 1 and 2.

1) An example is shown in Fig. 1. The rhythmical variations in the splenic volume were induced by an intravenous injection of $20 \mathrm{ml}$ of defibrinated blood. The arterial blood pressure began to rise immediately after the injection, which accompanied a transient fall in venous blood pressure, and thereafter the rhythmical undulations developed in the arterial pressure. The amplitude of the changes was $25 \mathrm{mmHg}$, and the durations of five undulations were 44, 45, 50, 50 and 52 seconds respectively, with an average of 48.2 seconds. The zeniths in the blood pressure tracing were almost synchronized with the nadirs of the splenic

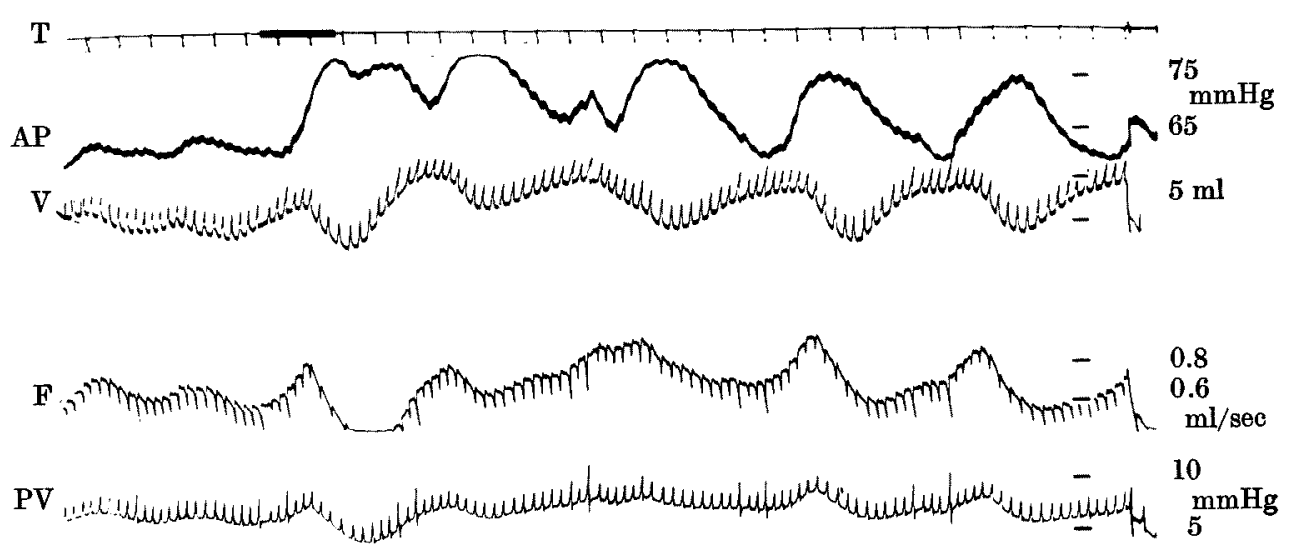

Fig. 1. Rhythmical changes in systemic pressure, splenic volume, splenic venous flow and splenic venous pressure after injection of defibrinated blood.

PA: Systemic blood pressure (carotid artery)

$\mathrm{V}$ : Splenic volume

F : Blood flow through the splenic vein

PV: Splenic venous pressure

$\mathrm{T}$ : Time in $10 \mathrm{sec}$

Signal indcates the time when $20 \mathrm{ml}$ of defibrinated blood were injected. 
volume tracing, but five splenic waves lagged 5, 3, 2, 8 and 1 seconds, respectively, behind the changes in blood pressure. The maximal variation in the splenic volume was $4.99 \mathrm{ml}$.

The changes in the blood flow through the splenic vein coincided with those in the splenic venous pressure, but there was a delay of $5,11,7$ and 7 seconds respectively at their peaks, when compared with peaks in the splenic volume changes.

The splenic venous pressure varied from 6.3 to $8.5 \mathrm{~mm} \mathrm{Hg}$. The rate of blood flow through the splenic vein during the time its rhythmical changes persisted was $0.6 \mathrm{ml}$ per minute greater than the control splenic venous flow (see Fig. 1).

2) Another example is shown in Fig. 2. The rhythmical contractions of the spleen were induced by temporarily clamping the splenic vein. The undulatory changes in the splenic volume and venous flow synchronized with the changes in the systemic arterial and splenic venous pressures, and were in the same direction. The durations of seven undulations of the systemic blood pressure in this figure were of $28,27,29,26.5,29,28$ and 29 seconds respectively, with an average of 28.1 seconds, the variations in the pressure ranging from 71 to $95 \mathrm{~mm}$ $\mathrm{Hg}$. The peaks of the splenic volume undulations lagged 4, 3, 1.5, 2.5, 2, 3.3 and 2.5 seconds, respectively, with an average of 2.7 seconds, behind the peaks in the systemic blood pressure.

The peaks in the undulations of the splenic blood flow and splenic venous

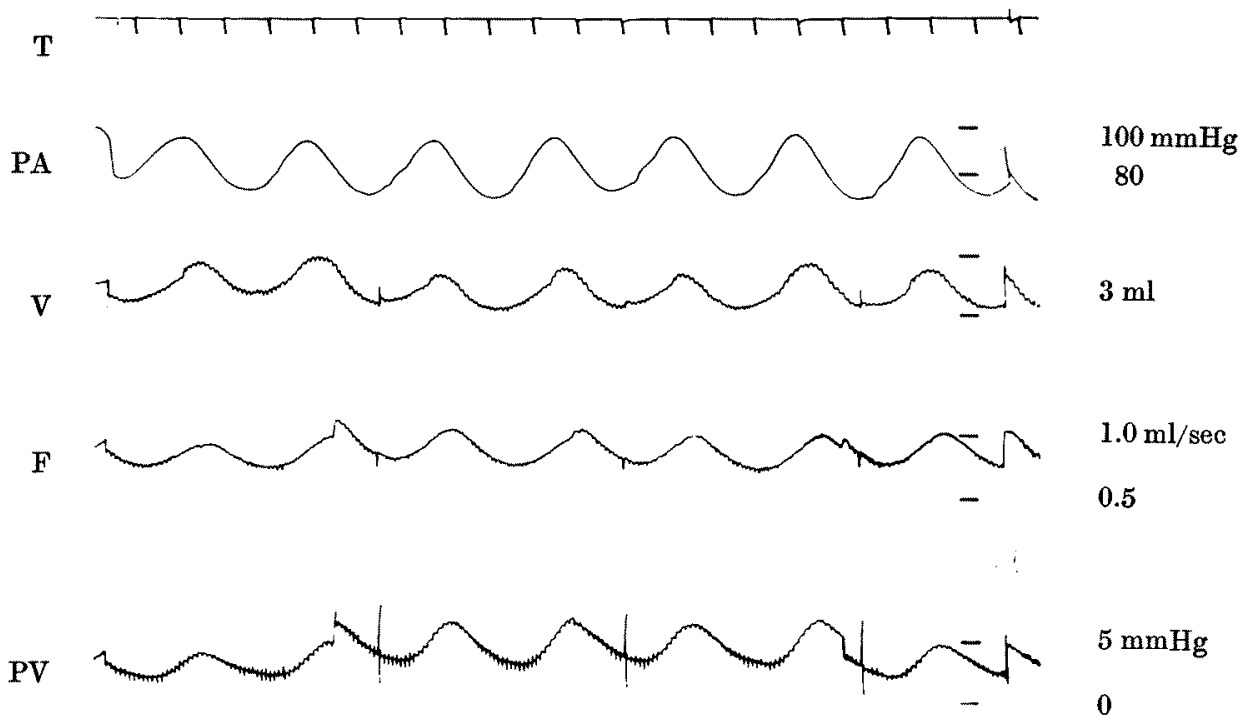

Fig. 2. Rhythmical changes in systemic pressure, splenic volume, splenic venous flow and splenic venous pressure about 17 minutes after the splenie vein was temporarily clamped.

The codes are the same as in Fig. 1 . 
pressure lagged 4, 3, 3, 4, 3.5, 2.5 and 2.5 seconds, respectively, with an average of 3.2 seconds behind the peaks in the undulations of the splenic volume. The variation in the splenic volume was $1.55 \mathrm{ml}$ and the rate of blood flow through the splenic vein,was $5.0 \mathrm{ml}$ per second. This was $2.1 \mathrm{ml}$ per second greater than the control'splenic venous flow. The venous pressure varied from 3.4 to $6.4 \mathrm{~mm} \mathrm{Hg}$, compared with its control level of 4.1 to $4.6 \mathrm{~mm} \mathrm{Hg}$.

\section{Discussion}

1) In Fig 1, the fluctuations in the splenic volume were in a direction opposite to those of the changes in the systemic blood pressure. This shows that the active contraction of the spleen causes a rise in the systemic blood pressure by increasing the peripheral resistance of the vascular system. ${ }^{4,5}$ It is conceivable that the contraction of the spleen pushes out its reserve blood into the splenic vein, bringing about a rise in the splenic venous pressure and an increase in the blood flow through the splenic vein. Thus, the pressure and flow in the splenic vein might be changed only passively by the variation of the splenic volume.

By contract, in Fig. 2 the undulations in the systemic blood pressure occurred in the same direction as those in the splenic volume, venous pressure and flow. Since the changes in the systemic blood pressure preceded the other changes, the splenic volume, venous pressure and flow might be influenced only passively by the arterial blood pressure changes.

However, there was a more or less considerable difference in the rhythm of changes between the systemic blood pressure on the one hand, and splenic volume and flow on the other. For example, during the period of 10 undulations with an average duration of 15.7 seconds in the systemic blood pressure, there were found 6 undulations with an average duration of 25.3 seconds in the splenic volume and flow.

Further, the undulatory changes in the splenic volume and flow were not always synchronous with each another. This asynchronism might be due, at least partly, to inherent rhythmical contractions of the portal vein itself.6,7 The undulatory changes in the hepatic volume might also participate in the production of the rhythm in the splenic venous flow. ${ }^{8}$

Occasionally, the nadirs of the undulations of the splenic blood flow were almost synchronous with the zeniths in the splenic volume and venous pressure fluctuations. The decrease in the splenic flow and the increase in the splenic volume and venous pressure coincided with the acceleration of the respiratory movements.

2) The rates of blood flow in the splenic vein in 11 cases in which the systemic blood pressure maintained a relatively constant level before and after its rhythmical undulations were induced are summarized in Table 1. 


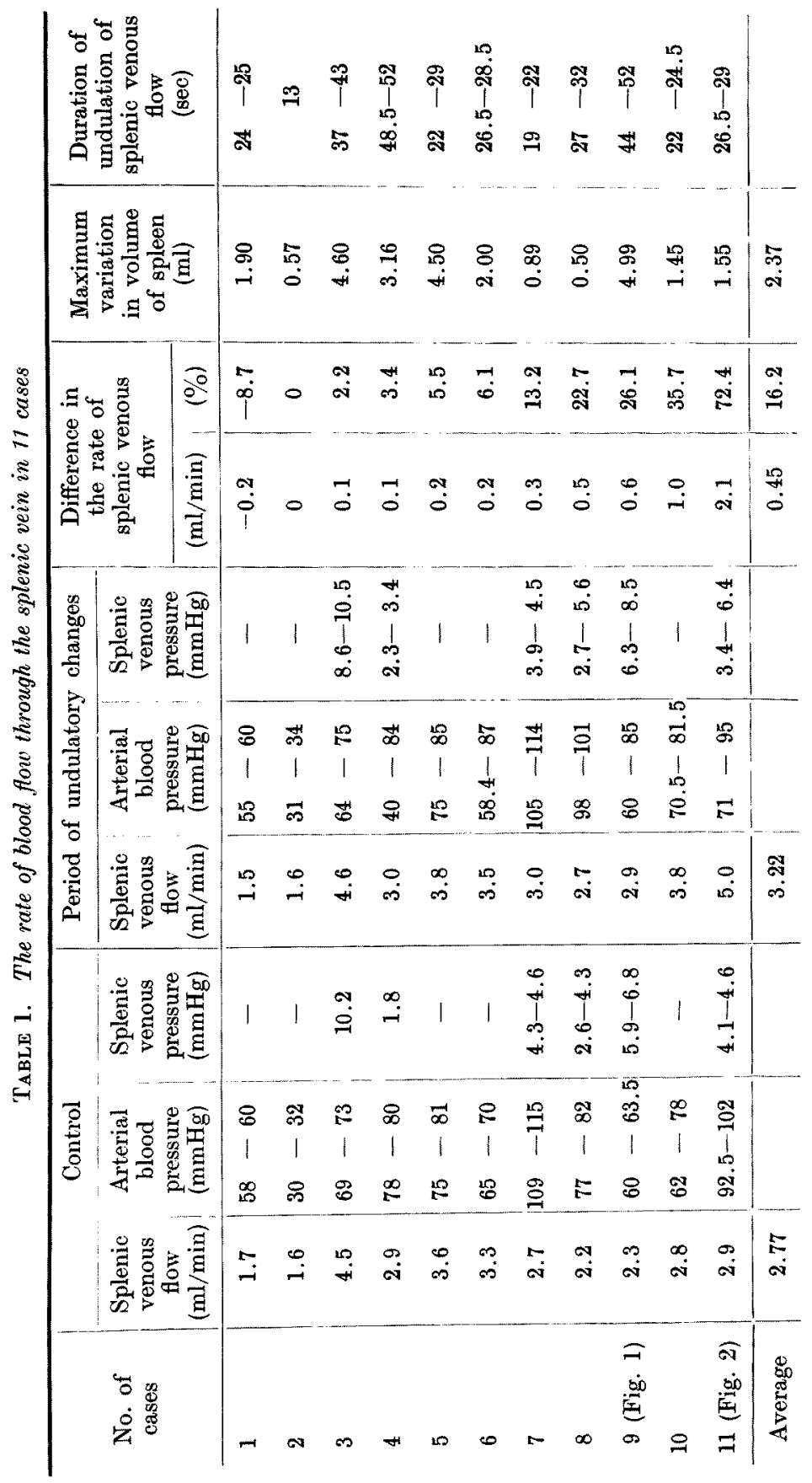


The increase in blood flow of the splenic vein was observed in 9 out of 11 cases. The control splenic venous flow averaged $2.77 \mathrm{ml}$ per minute in 11 cases, but increased to $3.22 \mathrm{ml}$ per minute during the period for which the undulatory changes persisted. This corresponded to $16.2 \%$ of the control splenic venous flow. The maximum variation in the splenic volume was $2.37 \mathrm{ml}$. These results suggest that sudden changes in the circulatory blood flow may produce rhythmical changes in the vascular tone and accelerate the redistribution of blood. It is quite possible that the rhythmical fluctuation in the splenic venous flow serves to increase the blood flow in the portal vein.

\section{Acknowledgment}

The authors wish to express their cordial thanks to Dr. Y. Nisimaru and Prof. M. Wada for their valuable advice and encouragement throughout this work.

\section{References}

1) Roy, C.S. The physiology and pathology of spleen. J. Physiol. (Lond.), 1881, 3, $203-228$.

2) Schäfer, A.E. \& Moore, B. On the contractility and innervation of the spleen. J. Physiol. (Lond.), 1896, 20, 1-50.

3) Barcroft, J. \& Nisimaru, Y. Undulatory changes of blood pressure. J. Physiol. (Lond.), 1932, 74, 311-320.

4) Semba, T., Fukuba, T. \& Kishi, Y. On the undulatory changes of the splenic venous pressure. J. physiol. Soc. Japan (Jap.), 1951, 13, 328-331.

5) Barcroft, J. \& Nisimaru, Y. Cause of rhythmical contraction of the spleen. J. Physiol. (Lond.), 1932, 74, $299-310$.

6) Naito, Y. On the rhythmical contraction of the portal vein of a dog. Hiroshima $J$. med. Sci., 1954, 3, 29-37.

7) Semba, T. Studies on the circulation of the portal vein. J. Hiroshima med. Ass. (Jap.), 1953, 6, 208-214.

8) Semba, T. \& Kishi, Y. Periodic changes in the liver volume and changes in the portal venous pressure. Jap. J. Physiol., 1952, 2, 296-302. 